\title{
Human Interface and Applications on IdeaBoard
}

\author{
Masaki Nakagawa, Tsuyoshi Oguni and Takashi Yoshino
}

\author{
Dept. of Computer Science \\ Tokyo Univ. of Agri. \& Tech. \\ Naka-cho 2-24-16, Koganei, Tokyo, 184, Japan \\ nakagawa@cc.tuat.ac.jp
}

\begin{abstract}
This paper describes a new style of human interface and several applications on an interactive dynamic whiteboard. If current GUIs are just expanded to the whiteboard size, the teacher would have to frequently move from side to side and stretch their hands from edge to edge, as well as the teacher's body would cover the board. In order to avoid these problems, operability from an arbitrary standing position of the teacher with a reasonable amount of hand movement, thus no hiding of the board by the body, and easy operations by electronic markers have been sought. Moreover, various applications have been prototyped. A programming education system is one of these, where the teacher writes a program on the board and can have it recognized, compiled and executed in front of the students. Another is an arithmetic education system where children write arithmetic equations on the board and the system answers them.
\end{abstract}

KEYWORDS pen interface, handwriting recognition, electronic whiteboard, direct pointing, GUI

\section{INTRODUCTION}

This research has been motivated from our experience in programming education.

So far, we have been teaching programming on a white/black board and telling students to try the programs that we have explained after the class. Next week, their answer is often that the programs have not worked. The reason is quite simple. Programs that we write on a white/black board are not always syntactically perfect and moreover students often misread them or miscopy them in their notebooks.

Then, the students ask us to lecture in a computer room. Now, they can try programming immediately. But, the problem is that they focus their attention on their PCs and do not pay attention to our explanations. It is very difficult to present intrinsic materials in a computer room.

On the other hand, the advantage with the white/black board is that we can focus the attention of students on our writing and present new materials while confirming their understanding by watching their faces. But, we cannot show the execution of programs in front of them.

Although practice on a PC is essential for each student to learn programming for themselves, to computerize the white/black board and provide it with the capability of showing program execution brings about new potential for programming education.

We decided to develop a system to combine the merit of the white/black board and that of computer processing as a part of pen interface research. The system may open the way for new applications other than programming education.

Human-Computer Interaction: INTERACT'97 S. Howard, J. Hammond \& G. Lindgaard (editors)

Published by Chapman \& Hall OIFIP 1997 
The Liveboard by XEROX is well-known as such a system (Elrod et al. 1992, Pedersen et al. 1993, Weiser 1991, 1993). The effect of capturing audience's attention and the benefit of recording and replaying handwriting has been reported. These observations apply to our system as well, but our system differs from the Liveboard in the following respects.

Firstly, the Liveboard and especially its applications developed at XEROX PARC avoid handwriting recognition except that for gestures (Pedersen 1993). On the other hand, we employ handwriting recognition, which is essential to compile and execute handwritten programs, to exploit the power of computing.

Secondly, the Liveboard uses gesture commands while we are not positive about employing them because of the reasons described below. We try to enhance the desktop GUI to make it suitable for the large interactive display and electronic markers.

The reason that the Liveboard employs them is that a simple expansion of the desktop GUI to the board size requires the user to push top left button as well as bottom right buttons, thus making the user's body cover the board. But, we try a different possibility.

Thirdly, the Liveboard seems to be oriented for meetings and presentations, but our system is mainly designed for educational use. To develop educational applications is an important part of our research.

Fourthly, the resolution of $640 \times 480$ and the brightness of the 67-inch color display of the Liveboard is not satisfactory. Even the updated resolution of $1024 \times 768$ of the Liveboard 3 is not good enough to be used as a whiteboard.

Enlarging a computer display and the sharing of it by participants makes collaboration easier by itself. To be used as an electronic whiteboard, however, brightness and resolution seem to be very important. Without a sufficient level of quality in these respects, the merit of the whiteboard will be reduced.

We considered employing the Liveboard, but decided to use a temporary hardware to develop software while preparing a new hardware described later. We call our system "IdeaBoard" (interactive, dynamic, electronic assistant board).

The basic concept of the IdeaBoard is not the enlargement of the computer display, but the coupling of the merits of a whiteboard with those of computer processing. This requires the very high resolution of a large display (more than $1600 \times 1280$ resolution of the 70-inch size) with electronic markers as well as a new interface design and applications which inherit the conventional and familiar interfaces from a whiteboard while exploiting the dynamic processing of contents by computing.

In this paper, we present user interfaces and applications on IdeaBoard.

\section{TEMPORARY HARDWARE}

The temporary hardware is composed of a 70 -inch tablet, an LCD projector and a Windows machine. Its appearance is shown in Figure 1. The display of the Windows machine is projected on the tablet from the LCD projector.

The tablet has a size of $1,331.1 \times 998.4 \mathrm{~mm}$ and approximately $4,000 \times 3,000$ resolution with 3 cordless markers and an oval-shaped eraser which are all battery free. A marker sends its position and touched/ untouched information which corresponds to the mouse's left button on the PC, but it is not equipped with a switch corresponding to the mouse's right button. The oval-shaped eraser has two point-indicators so that the system can sense the position and orientation of the eraser. Each pen or eraser sends its own identification code so that its role can be changed by software. One of the hardware limitations is that multiple pens and the eraser cannot be used at the same time.

The LCD projector has a resolution of $640 \times 480$, a brightness of 250 lumen and $16 \mathrm{M}$ colors, which restricts the specification of the temporary IdeaBoard.

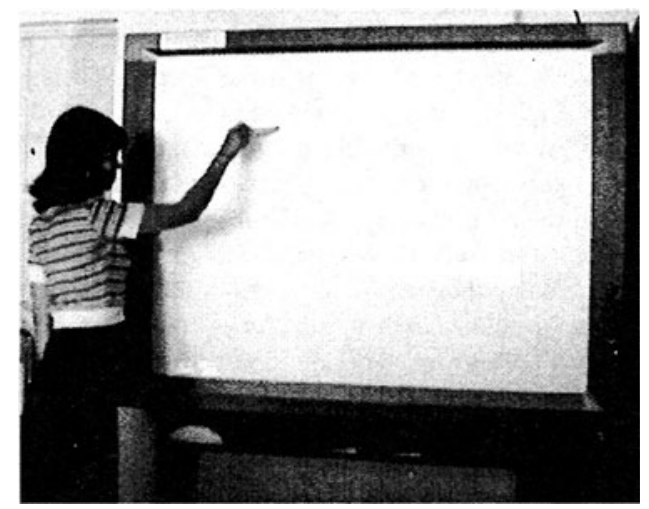

Figure 1. The temporary IdeaBoard in use. 


\section{DESIGNING THE IdeaBoard UI}

\subsection{UI for a Large Surface with Markers}

If we just expand the usual GUI for the desktop machine to the whiteboard size, problems occur in its usability. A teacher has to move from side to side, stretch hands from button to button, which not only makes the teacher practice gymnastics but often hides the board from the students. Often, operations too minute are also required which are difficult for the hand of a standing person. Moreover, double tapping with a marker is not as easy as with a mouse. Concurrent use of markers and a keyboard is far more difficult than that of a mouse and a keyboard because their manipulation scales are so different.

In order to design the interfaces, the size of the board, its vertical position, operability by markers, body actions by a teacher as well as the consistency with the desktop GUI must be taken into consideration.

\subsection{Gestures Considered Harmful}

Gestures have been an attraction of pen interfaces. The Liveboard has also employed gestures. We are reluctant, however, to employ gestures because of the following reasons:

(1) Design of gestures is very difficult. Often, gestures are difficult to remember and easy to forget.

(2) Gestures have simple shapes and simple shapes are hard for machines to recognize.

(3) There is little context to augment gesture recognition.

(4) Mode change for inputting gestures is troublesome and easy to forget.

(5) Misrecognition of a gesture or forgotten mode change yields an abrupt and unexpected result which must be canceled. It not only interrupts lectures or presentations very badly but also shocks the teacher or presenter.

(6) Because of the above problems, users fear that gestures might be misinterpreted and may hesitate to use gestures.

We think that pattern recognition can be more usefully employed for contents rather than for commands.

\subsection{Extension of the Desktop GUI}

We are now very familiar with the desktop GUI. It has apparent advantages against the old style of user interfaces as well as being refined to be accepted from a majority of users.

Even if a simple expansion of the desktop GUI to the whiteboard size is not usable, to employ or extend its styles or elements in a form that they are consistent with the desktop while enhancing the usability of the IdeaBoard can still be effective.

\subsection{Guidelines}

In the process of reviewing the desktop GUI, we have set the following guidelines:

(1) Operability from any standing position

To operate IdeaBoard without crossing the surface, gestures or pop-up menus can be considered. In order to invoke the pop-up menus, a gesture or an extra action is required. We tried to solve the problem by a different method with the temporary hardware.

(2) Operability by body size movement

Too large a movement, required for a user to operate the IdeaBoard, is hard and ends up hiding the board, but too minute a movement is also difficult for the standing user. Body size movement with direct pointing and manipulation is not only natural to the user but also appealing to the audience and it navigates their focus of attention.

(3) Easy operability by a marker

To keep pushing a marker horizontally at one point on the vertical board is hard for a person. His/her hand becomes tired in trying to remain steady. Compared with this, dragging (drawing) a marker is easier and more direct for the audience so that its employment is considered.

(4) Natural extension of the desktop GUI

We try not to create new styles or elements of GUI as far as those of the desktop GUI can be effectively employed. When we need to extend or modify them, we try to be as consistent as possible with the desktop so that the users do not feel a big difference.

(5) Smooth movement of displayed objects

Very smooth and continuous movement of displayed objects such as shown in Pad (Perlin 1993) seems more important for the shared large display. We try to execute the actions on the board as smoothly as possible so that the audience can keep their focus of attention 
without being annoyed by the sudden change of contents.

\section{ELEMENTS OF THE IdeaBoard UI}

\subsection{Scroll}

Being different from the common scrolling by tapping or dragging a button or a bar at a fixed position, the IdeaBoard enables a user to scroll in any direction from any standing position.

We place the scroll bar around the board as shown in Figure 2. If you touch a marker to any point in the scroll bar and drag it in any direction, then the window is scrolled in that direction. You can scroll from any standing position and you do not have to move to the scroll button and keep pushing it. If you move the marker $(x, y)$ after touching the scroll bar, then the window is scrolled by $(k x, k y)$ where $k$ is determined by the velocity of the marker movement.

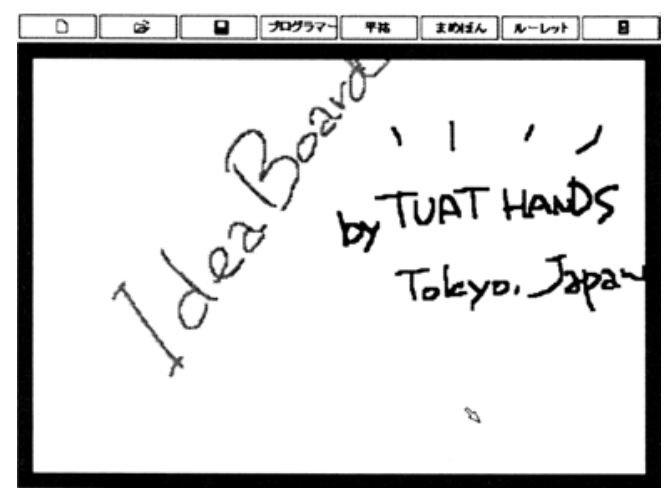

Figure 2. Scrolling from any point in the window frame.

\subsection{Window Movement}

We propose the following method for window movement. If you touch a marker to the title bar and drag it to $(x, y)$, the window is moved to $(k x, k y)$. The coefficient $k$ is adjusted so that the window can be moved around in the display if you stretch your hand with the marker from any standing position.

By drawing a line between $(x, y)$ and $(k x, k y)$, you can easily observe what and how you are moving. Its appearance has already been shown in Figure 1.

\subsection{Tool Bar}

We propose a tool bar as shown in Figure 3. Function buttons in the tool bar extend or shrink as the window is widened or narrowed. One might have to stretch one's hand, but even the most distant function button is extended as the window is widened so that one is unlikely to hide the window.

(a)

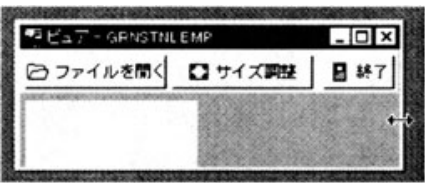

(b)

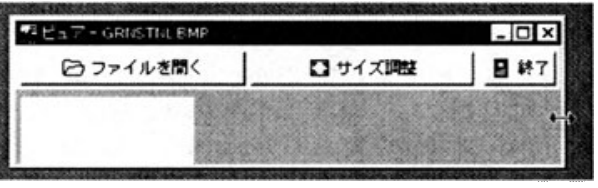

Figure 3. Extensible tool bar (from a to b).

\subsection{Counter}

Common counters are as shown in Figure 4 (a). If you push or keep pushing the up/down button, the counter is increased/decreased.

We propose a counter as shown in Figure 4 (b). The up button and down button are merged to provide a bigger button. If you touch a marker to any point in the button and drags up or down, the counter is increased or decreased in proportion to the amount of the marker's movement. This dispenses with the need to keep touching the marker or touch it many times at the same point.

(a)

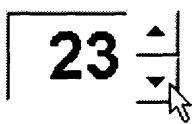

(b)

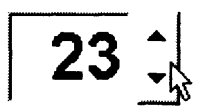

Figure 4 . Counter setting by dragging.

\subsection{Tab}

Tab is used to turn a page. We propose the following type of tab as shown in Figure 5. When a tab is shown on the right side in the page, tapping at the left side causes the tab to be displayed there. When it is shown on the left, tapping at the right brings the tab to the right. 
A user need not move to the other side to tap the tab.

Moreover, touching a tab item with the marker and dragging it up or down causes a jump to upper or lower tab items according to the amount of the marker's movement. This dispenses with careful tapping to the right destination since the destination can be reached by just dragging the marker up or down while confirming the visual feedback.

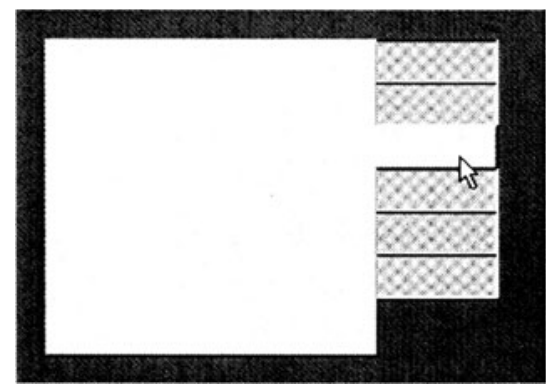

Figure 5. A proposed tab.

\subsection{Turning Pages}

We propose page turning by dragging the marker to the direction that one wants to go rather than pushing page turning buttons. Figure 6 shows an example. When pages are just for viewing, touching the marker at any position and dragging to right/left causes the page to be turned to the right/left. One need not tap the marker in specified positions. Whether you are touching the marker on the right page or the left page is not important. Page turning direction is just determined by the direction of dragging. Thus, a teacher can turn the page to either right or left direction whether standing at right or left of the board. When the pen is dragged by $x$, the page is turned by $k x$. The coefficient $k$ is adjusted so that amount of hand movement is appropriate.

\section{APPLICATIONS ON THE IdeaBoard}

Applications on the IdeaBoard employ handwriting recognition when necessary, but they do not blindly try to recognize handwriting whatever and whenever it is. The following applications have been prototyped.

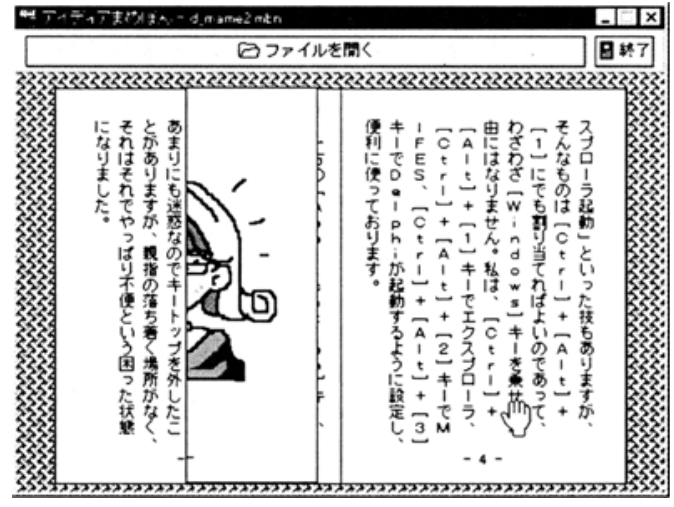

Figure 6. Page turning by dragging.

\subsection{Programming Education}

A teacher writes a program, explains it, then makes the system recognize and execute it. Here, program recognition is not for program input but for programming education and demonstration.

Figures 7, 8, and 9 show a handwritten program, the result of its recognition using the context of a $C$ program, and the result of its execution, respectively.

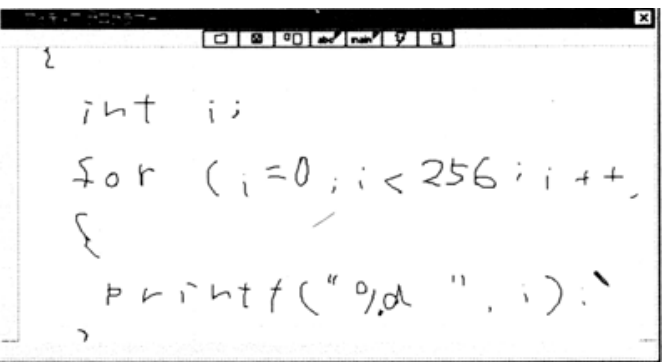

Figure 7. A handwritten program.

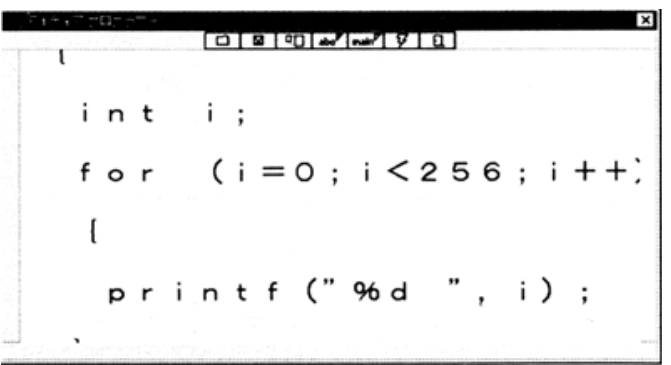

Figure 8. Result of program recognition. 


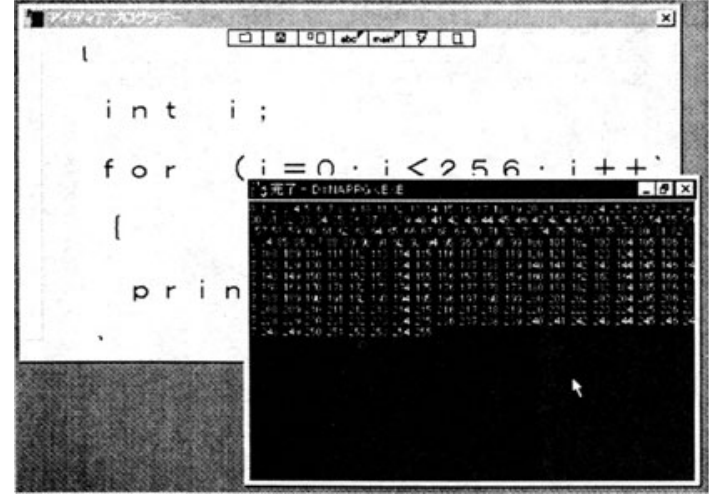

Figure 9. Result of program execution.

Here, we follow the lazy recognition scheme which delays the display of recognition until needed. Lazy recognition also provides an easier structure to employ context processing (Nakagawa et al. 1993a, 1993b).

When a teacher is writing a program and explaining it, machine recognition is not only unnecessary for the teacher and students, but it is even worse than when no recognition is employed. Recognition immediately after a pattern is written causes interruption because of the correction of incorrect recognition and the verification of recognition even if a pattern is correctly recognized.

When the teacher wants to show the execution of a handwritten program, however, program recognition is highly convenient and effective. Although the teacher might have to correct some misrecognitions, it is worth the trouble since the machine can show the execution of the program that the teacher has just written in front of the students.

Editing functions for space insertion and deletion of characters and lines can be triggered by tapping a marker between lines and dragging right or left (blank insertion or character deletion ) or dragging down or up (blank line insertion or line deletion). They are executed smoothly.

Smoothness has been sought to realize move and copy functions as well. If you tap a marker between lines and wait for a certain period, then the color of the upper line is reversed. If you drag the marker up/down without detaching it, the color of more upper/lower lines are reversed. This shows that lines of text have been selected. Then, when you tap the marker at some place outside the reversed area, the selected lines are copied. On the other hand, if you tap the reversed area again, the selected lines are drawn into the marker smoothly. Then, when you tap the maker at some place, the saved lines are released there.

\subsection{Arithmetic Education}

Children write arithmetic equations on the board and the system answers whether they are correct or not. They can share calculations with others, teachers and the system. The same editing functions as the programming education system are provided. Figure 10 shows the IdeaBoard with this application running.

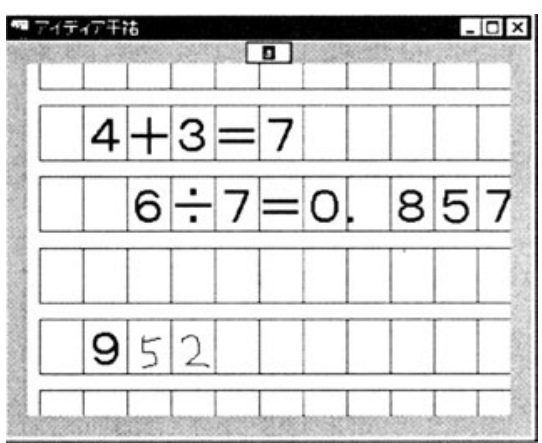

Figure 10. Arithmetic education on IdeaBoard.

\subsection{WWW Browser}

The IdeaBoard is expected to be useful for visiting electronic museums and libraries where a teacher can focus the attention of students on the shared picture on the IdeaBoard.

This application also employs the enlargement of hand movement. A distant object can be pointed to by a pointer which can be stretched from any point in the window frame as shown in Figure 11 . You only have to touch the marker anywhere in the frame and drag it to the direction of the destination. Your hand movement is enlarged so that the pointer head can point to the destination without requiring you to move to the destination with your body hiding the board. With this pointer, pointing to an anchor then detaching the marker causes a jump to its linked page. URL addresses or key words for search can be input through the front end of a handwriting recognizer. 


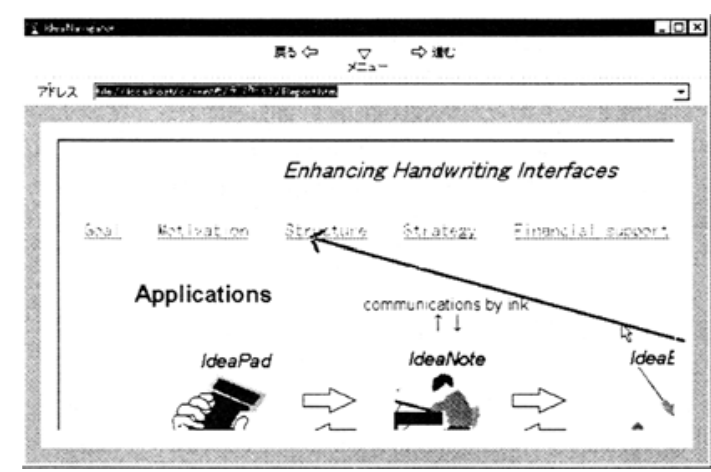

Figure 11. WWW Browser on IdeaBoard.

\subsection{Other Applications}

In addition to the above, handwriting training where a student writes Kanji and the system evaluates the correctness of handwriting using the recognition engine, and brainstorming support where idea cards are arranged on the board and they are recognized and summarized into a document are the major applications proposed to be run on the IdeaBoard. For these applications, handwriting recognition is useful if it has more than a certain level of quality and it can be upgraded by context processing. We have developed a highly robust and customizable character recognition engine (Nakagawa et al. 1996a, 1996b).

The difference in the stance towards pattern recognition thus described makes the IdeaBoard system different from the Liveboard system.

\section{FUTURE WORK}

\subsection{Enhancement of UI and Applications}

Following the current design principle, some more UI elements would be required to run some other applications. Moreover, each of the current applications must be elaborated through the iterative design method. Effectiveness of the IdeaBoard must be evaluated through the expansion of its scope as well as the enhancement of each application.

A better way to invoke multiple applications must be considered. When switching from one application to another, relocation and resizing of windows are needed as on a desktop environment. The problem is that they are more troublesome on the large board than on the desktop environment. Seamless combination of multiple applications must be studied.

We decided not to employ gestures since their misrecognition disrupts lectures and presentations in unexpected ways. There is another serious problem with the gestures, which is the mode change required to input them. It is troublesome if a teacher must touch some button every time to change the mode of the pen. Moreover, the teacher might forget to change the mode with the result that an ink pattern might be misinterpreted as a gesture or vice versa, which all must be canceled.

Nevertheless, a limited number of gestures are still appealing to the pen UI design. With an appropriate design, a robust recognition method, good interface design that avoids mode selection, gestures might be able to provide expected benefits.

\subsection{Development of a New Hardware}

The hardware that we are currently using is unsatisfactory. Front projection is dazzling to the teacher's eyes and its display is hidden by the teacher's body. A resolution of $640 \times 480$ is too coarse since 1 pixel is about $2 \mathrm{~mm} \times 2 \mathrm{~mm}$ in size when enlarged to the 70 -inch size. When we write a character of size of 20 $\mathrm{mm} \times 20 \mathrm{~mm}$, it has only a resolution of $10 \times 10$ dots. If it is a English font, it may be legible. For handwritten Kanji characters, however, the resolution is too poor for them to be displayed as they are written. Therefore, we cannot write Japanese text or mathematical formulas as we can on a whiteboard. It is also very difficult to carry out brainstorming while displaying tens or hundreds of electronic idea cards which have been written on PDAs. Brightness of the display is also a problem. If a class room has to be dark like a theater, the demerits overwhelm than the merits. The display must be usable in a well-lighted environment so that students can see the teacher's face and actions as well as the screen. Then, both the real explanation by the teacher and the virtual contents of the display are merged on the students' retinas. With the limited hardware, the possibility of software will be restricted and the evaluation of the system is destined to be poor.

We started to make a new hardware based on a rearprojection 70-inch High Vision TV which has a 680 $\mathrm{cd} / \mathrm{m}^{2}$ brightness, a $180: 1$ contrast rate and a $1600 \mathrm{x}$ 
1280 resolution. With better hardware, various possibilities for software can be studied and issues will be clarified without putting the blame on hardware.

\subsection{Total System for Pen Interface}

Pen is a scalable interface unlike mouse or keyboard, i.e., it can be used commonly between PDAs, desktop display integrated tablets and large interactive boards. The total system is one in which you write down your idea memos on a PDA wherever you might be, then download them to a desktop environment and have them recognized when necessary, or you prepare materials for a lecture on the desktop and carry out the actual lecture on the IdeaBoard. The live lectures can be recorded and reviewed on a desktop or PDA. The lazy recognition scheme is employed throughout the total system.

Although the total architecture, composed of small, medium-size, and large pen input devices, is again similar to the ubiquitous computing at PARC (Weiser 1991, 1993), our aim is to provide the power of computing to our behaviors of handwriting which are made on media including various sizes of papers, notebooks and a white/black board. In contrast to this, the PARC's research is oriented more for collaboration where the small device (ParcTab) is mainly used to locate where the user is rather than for writing down memos.

\section{CONCLUSION}

This paper has described the IdeaBoard interface and application prototypes which are expected to succeed the merit of the white/black board as well as to provide users with computing powers to manipulate and interpret handwriting for education.

Enhancement of the IdeaBoard environment based on the evaluation of the prototypes as well as the development of the better hardware are being carried out.

\section{ACKNOWLEDGMENT}

This work is being supported by the Advanced Software Enrichment Project of IPA under MITI, Japan. We would also like to thank Hitachi, Hitachi Software and Fujitsu for their collaboration.

\section{REFERENCES}

Elrod, S., Bruce, R., Gold, R., Goldberg, D., Halasz, F., Janssen, W., Lee, D., McCall, K., Pedersen, E., Pier, K., Tang, J. and Welch, B. (1992) Liveboard: A Large Interactive Display Supporting Group Meetings, Presentations and Remote Collaboration. Proc. CHI'92, 599-607.

Nakagawa, M., Machii, K., Kato, N. and Souya, T. (1993a) Lazy Recognition as a Principle of Pen Interfaces, INTERCHI'93 Adjunct Proc. 89-90.

Nakagawa, M., Kato, N., Machii, K. and Souya, T (1993b) Principles of pen interface design for creative work, Proc. ICDAR'93, 718-721.

Nakagawa, M. and Tu, L.V. (1996a) Structural Learning of Character Patterns for On-line Recognition of Handwritten Japanese Characters, in Advances in Structural and Syntactic Pattern Recognition (eds. P. Perner, P. Wang and A. Rosenfeld), Springer-Verlag, 180-188.

Nakagawa, M., Akiyama, K., Tu, L.V., Homma, A. and Higashiyama, T. (1996b) Robust and Highly Customizable Recognition of On-line Handwritten Japanese Characters, Proc. 13th ICPR, III, 269-273.

Pedersen, E.R., McCall, K., Moran, T.P., Halasz, F.G. (1993) Tivoli: An Electronic Whiteboard for Informal Workgroup Meetings, Proc. INTERCHI'93, 391-398.

Perlin, K. and Fox, D. Pad: An Alternative Approach to the Computer Interface, Proc. 1993 ACM SIGGRAPH Conference, 57-64.

Weiser, M. (1991) The Computer for the 21st Century, Scientific American, 94-104.

Weiser, M. (1993) Some Computer Science Issues in Ubiquitous Computing, $C$. $A C M, 36,7,74-84$. 